\title{
X-ray Microanalysis of Porous Materials Using Monte Carlo Simulations
}

\author{
D. Poirier*, R. Gauvin* and R.A.L. Drew* \\ *Department of Mining, Metals and Materials Engineering, McGill University, Wong Building, \\ 3610, University street, Montréal, Canada, H3A 2B2
}

Quantitative X-ray micro-analysis models, such as ZAF or phi-ro-Z methods, are based on solid-flatpolished specimens. This limits their use in various domains where porous materials are studied, such as powder metallurgy, catalysts, foams, etc. Previous experimental studies have shown that an increase in porosity leads to a deficit in X-ray emission, but the mechanisms responsible for this decrease are unclear. In order to better understand the effects of porosity on quantitative microanalysis, a new approach using Monte Carlo simulations was developed [1]. In this approach, the X-ray emissions from porous materials were modeled using a random distribution of pores in the simulated materials. This paper presents some of the results obtained with this model and compares them with experimental results.

Single scattering Monte Carlo approach was used to simulate 20000 electron trajectories. At each elastic collision, the probability to encounter a pore was computed. If the electron was meeting a pore, then the effective distance traveled through the pore was randomly computed and added to the total distance computed between collisions. X-ray intensity was then calculated using phi-ro-Z technique with the absorption of photons computed individually for each generated X-rays with the probability that a pore is encountered. Simulations of silver at an incident beam energy of $20 \mathrm{keV}$ were then performed varying porosity conditions.

Figure 1 shows the variation of the backscattering coefficient according to the radius of the pores and for porosity values from 0 to 0.5 . Porosity seems to favor backscattering and the effect is maximum for pores of around 1micron of radius. This size is similar to the diameter of the interaction volume in bulk silver at $20 \mathrm{keV}$. Therefore, there is a good probability that an incident electron that does not encounter a pore can do so as it travels back to the surface, increasing the backscattering coefficient.

No variation of the $\mathrm{K}$ ratios of silver, defined as the net intensity emitted from a porous material divided by the intensity emitted by a bulk material of the same composition, were observed during the various simulations. In order to account for this X-ray deficit with porosity observed experimentally, a mechanism of extra energy loss by the diffusion of electrons by surface plasmons generated at the pores-solid interface was included in the model. The simulated $\mathrm{K}$ ratios are compared with experimental data obtained previously by R.E. Lakis et al [2] in Figure 2. The surface plasmons energy of silver is around $4 \mathrm{eV}$ from experimental measurements [3]. Porosities with radiis as low as $1 \mathrm{~nm}$ had to be modeled in order to see an effect on the $\mathrm{K}$ ratios with this value for energy loss. When the modeled pore radius was fixed at 1 micron to correspond to the experimental value, the loss of energy had to be increased to the unrealistic value of $1000 \mathrm{eV}$ to see an effect on $\mathrm{K}$ ratios.

Therefore, energy loss by the diffusion of electrons by surface plasmons generated at the pores can not explain the deficit of X-rays emission with porosity and another explanation has to be found. 


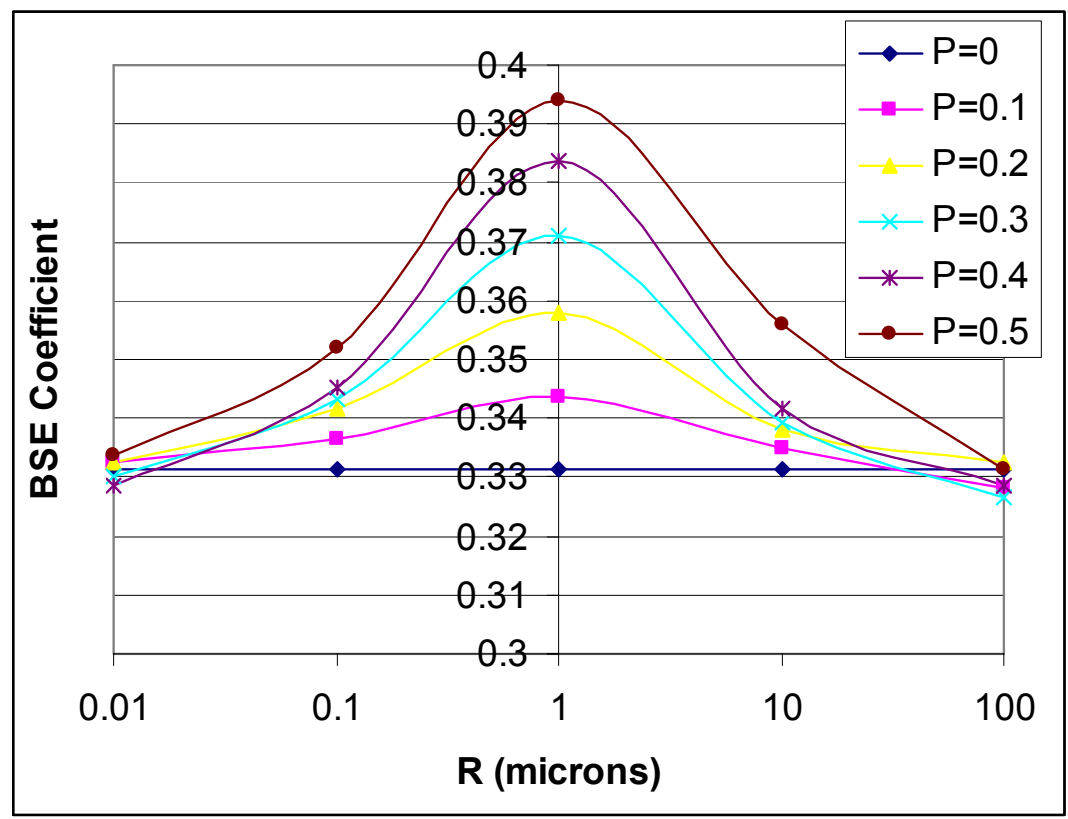

Fig. 1 Backscattering coefficient as a function of the pore radius, $\mathrm{R}$, and for different porosity in $\mathrm{Ag}$ at an incident electron energy equal to $20 \mathrm{keV}$.

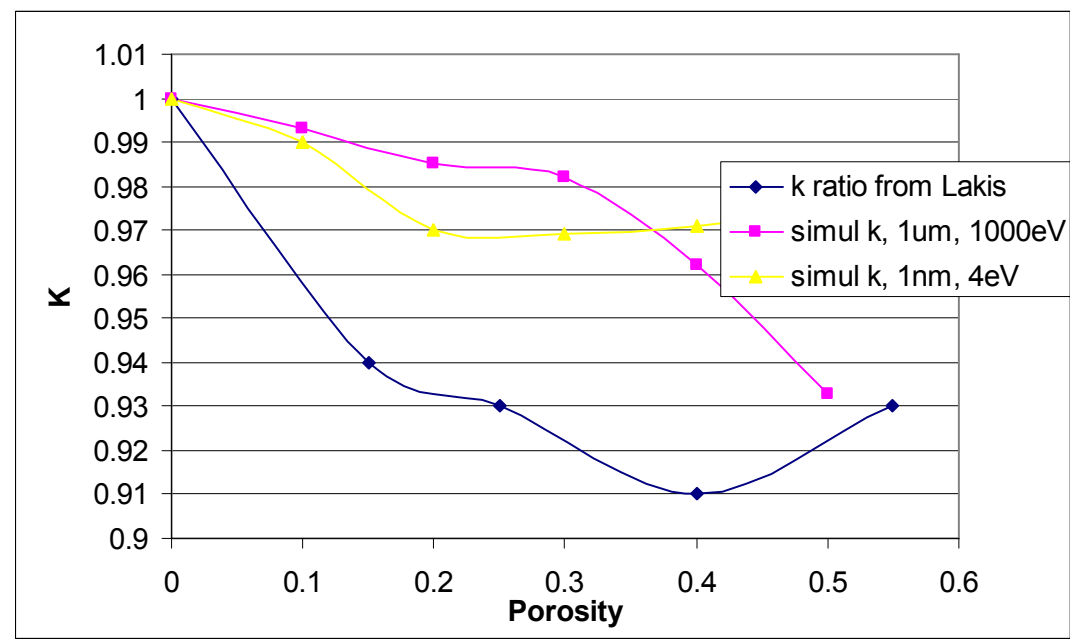

Fig. $2 \mathrm{~K}$ ratios of the Ag La line versus porosity in silver at incident electron energy equal to $20 \mathrm{keV}$. Simulated values with pores radius of $1 \mathrm{~nm}$ and 1 micron and an energy loss of $4 \mathrm{eV}$ and $1000 \mathrm{eV}$ respectively. Experimental values are taken from [2].

\section{References}

[1] R. Gauvin, Surf. Interface Anal. 37 (2005) 875.

[2] R. E. Lakis et al., Proc. 50th Annual Meeting of the Electron Microscopy society of America (1992) 1660.

[3] O. Millo et al., Phys. Rev. 39 (1989) 1006. 